\title{
hyperkeratotic uremic stomatitis revealing decompensation of chronic kidney disease
}

eya moussaoui ${ }^{1}$, wahbi ben salha ${ }^{1}$, Baderedine Sriha $^{2}$, lamia oualha ${ }^{1}$, and nabiha douki ${ }^{1}$

${ }^{1}$ University of Monastir Faculty of Dental Medicine of Monastir

${ }^{2}$ Farhat Hached University Hospital of Sousse

December 28, 2021

\begin{abstract}
Uremic stomatitis is a rare manifestation associated with longstanding uremia in chronic renal failure patients. Its evolution is favorable with earlier institution of renal replacement therapy. We report a case of hyperkeratotic uremic stomatitis that revealed decompensation of chronic kidney disease.
\end{abstract}

Title: hyperkeratotic uremic stomatitis revealing decompensation of chronic kidney disease Moussaoui Eya (1,2); Ben Salha Wahbi $(1,2)$; Badreddine sriha (3) ;Lamia Oualha (1) Nabiha Douki $(1,2)$

1: Department of dental medecine, SAHLOUL Hospital (Sousse), Dental Faculty of Monastir, University of Monastir, Tunisia.

2. Laboratory of oral health and maxillofacial rehabilitation (LR12ES11), University of Monastir, Tunisia.

3. Cytology and pathological anatomy Department, FARHAT HACHED Hospital (Sousse), University of Sousse, Tunisia.

\section{Corresponding author:}

Eya MOUSSAOUI : mail address: eyamouss@gmail.com Address: SAHLOUL Hospital, Department of dental medicine, Sousse, Tunisia.

Assistant professor at the department of dental medicine, Department of dental medecine, SAHLOUL Hospital (Sousse), University of Monastir.

Co- authors:

Ben Salha Wahbi: mail address: bensalhawahbi@gmail.com

Resident at the department of dental medicine, Department of dental medecine, SAHLOUL Hospital (Sousse), University of Monastir.

Badreddine sriha: mail address: badrisriha@gmail.com

Professor at the department of cytology and pathological anatomy,

Cytology and pathological anatomy Department, FARHAT HACHED Hospital (Sousse), University of Sousse, Tunisia.

*Lamia OUALHA: mail address: lamia.oualha@gmail.com Address: SAHLOUL Hospital, Department of dental medecine, Sousse, Tunisia. 
Professor at the department of dental medicine, SAHLOUL Hospital (Sousse), University of Monastir.

*Nabiha DOUKI : mail address: nabiha.douki@gmail.com Address: SAHLOUL Hospital, Department of dental medecine, Sousse, Tunisia.

Professor and head of department of dental medicine, SAHLOUL Hospital (Sousse), University of Monastir.

\section{Patient consent:}

I confirm that written patient consent has been signed and collected in accordance with the journal's patient consent policy.

\section{Author contribution:}

Eya moussaoui : aquisation and analysis of data and drafting the manuscript

Ben salha wahbi: data collection and interpretation

Badreddine sriha: collection and interpretation of data

Ouaha lamia: revising the manuscript for important intellectual content

Nabiha douki: conception and design and revising the manuscript

Key clinical message: The hyperkeratotic uremic stomatitis should be suspected by the dentist every time the context of end stage renal disease is present. The high blood uremia, in absence of other possible etiologies will confirm the diagnosis. After decreasing blood urea level by hemodialysis, lesions will disappear.

Keywords: uremic stomatitis, uremia, oral hyperkeratosis, chronic renal failure, hemodialysis.

A 72- year- old female patient was referred by her dermatologist to the department of dental medicine. She presented with burning oral lesions and dysguesia. The medical history was significant for chronic kidney failure.

The Intraoral examination revealed adherent white plaque on the floor of the mouth, the labial and buccal mucosa, the dorsal surface and the lateral borders of the tongue (Fig 1). This plaque could not be scraped off.

In view of her medical history and the extensive nature of the oral lesions, a diagnosis of hyperkeratotic uremic stomatitis was made. A renal biochemical profile was requested. It showed an increased level of blood urea $(360 \mathrm{mg} / \mathrm{dl})$

Biopsies of the lesions showed a hyperplasic epithelium and sloughing of superficial keratin layers with minimal underlying inflammatory infiltrate, thus excluding other oral disorders (Fig2).

The patient was referred to the department of nephrology for further investigation and treatment. After 4 sessions of hemodialysis, the blood urea nitrogen level (BUN) decreased and the white lesions were completely eliminated.

Uremic stomatitis is a rare manifestation associated with longstanding uremia in chronic renal failure patients [1]. Its evolution is favorable with earlier institution of renal replacement therapy [2].

[1] Barie E. De la stomatite urémique. Arch Gen Med 1889;2:415-32.

[2] Liao C-Y, Wu C-C, Chu P-L. Uremic stomatitis. QJM 2017;110:247-8. 

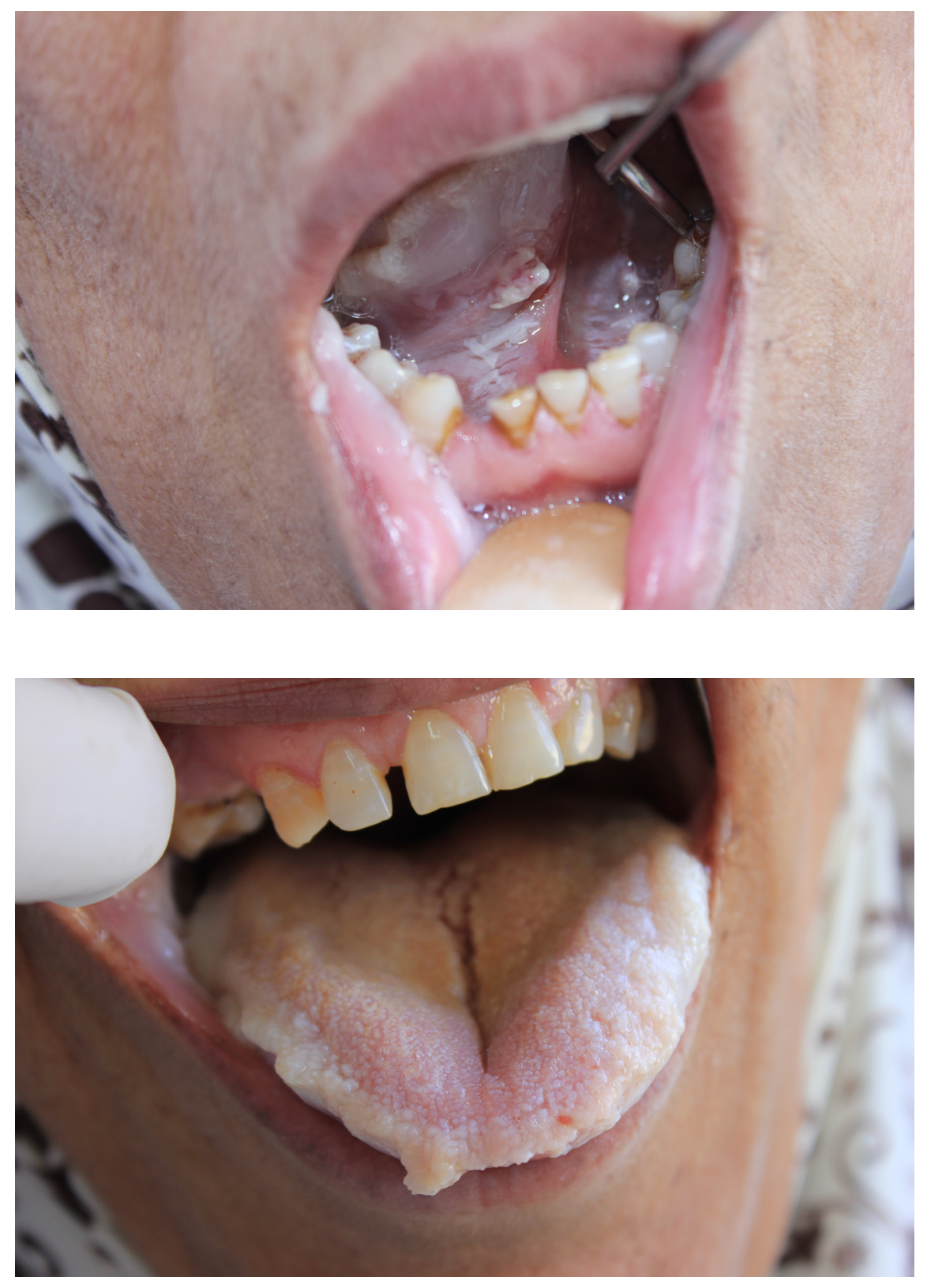

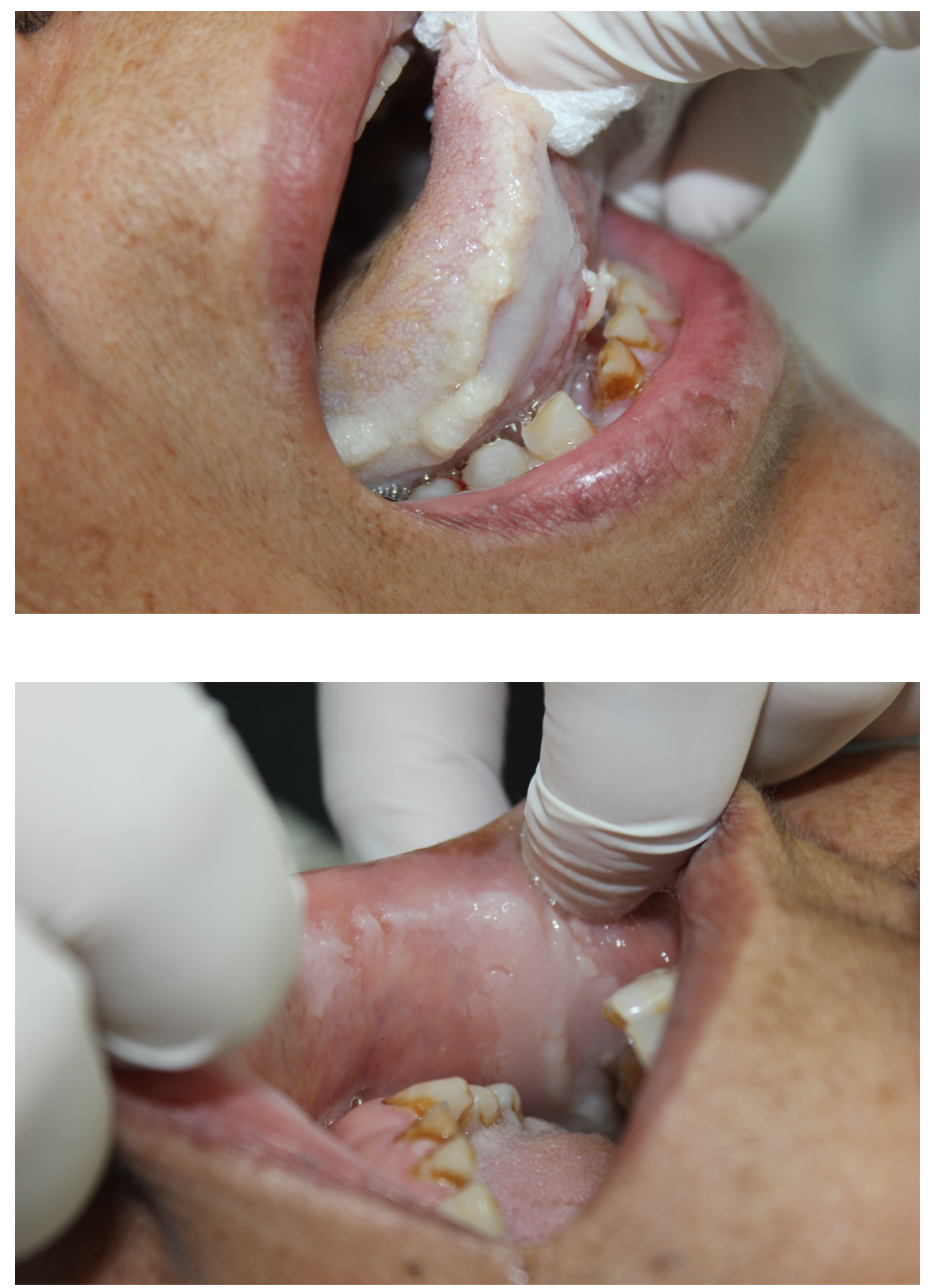

Conflicts of interest: 

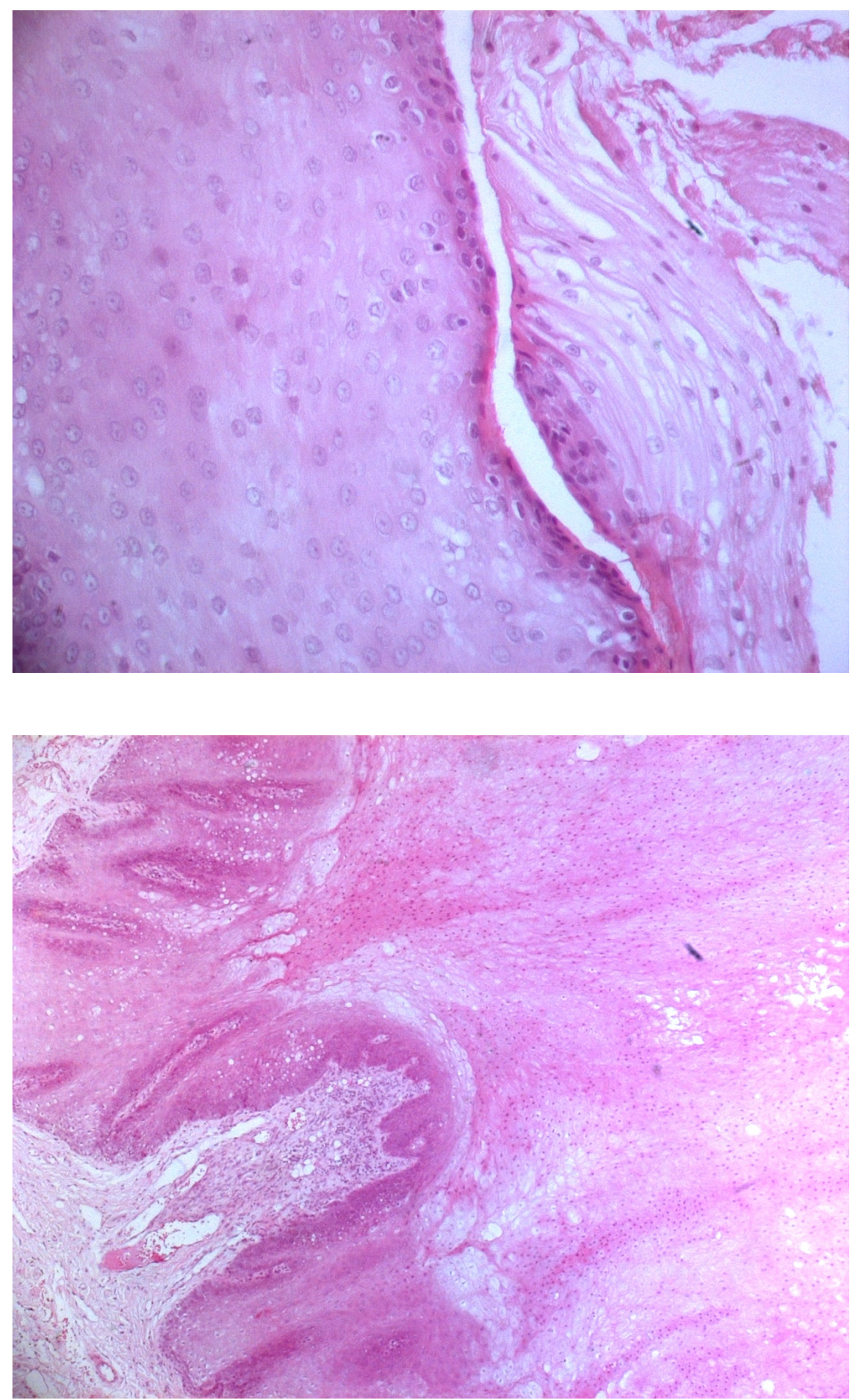


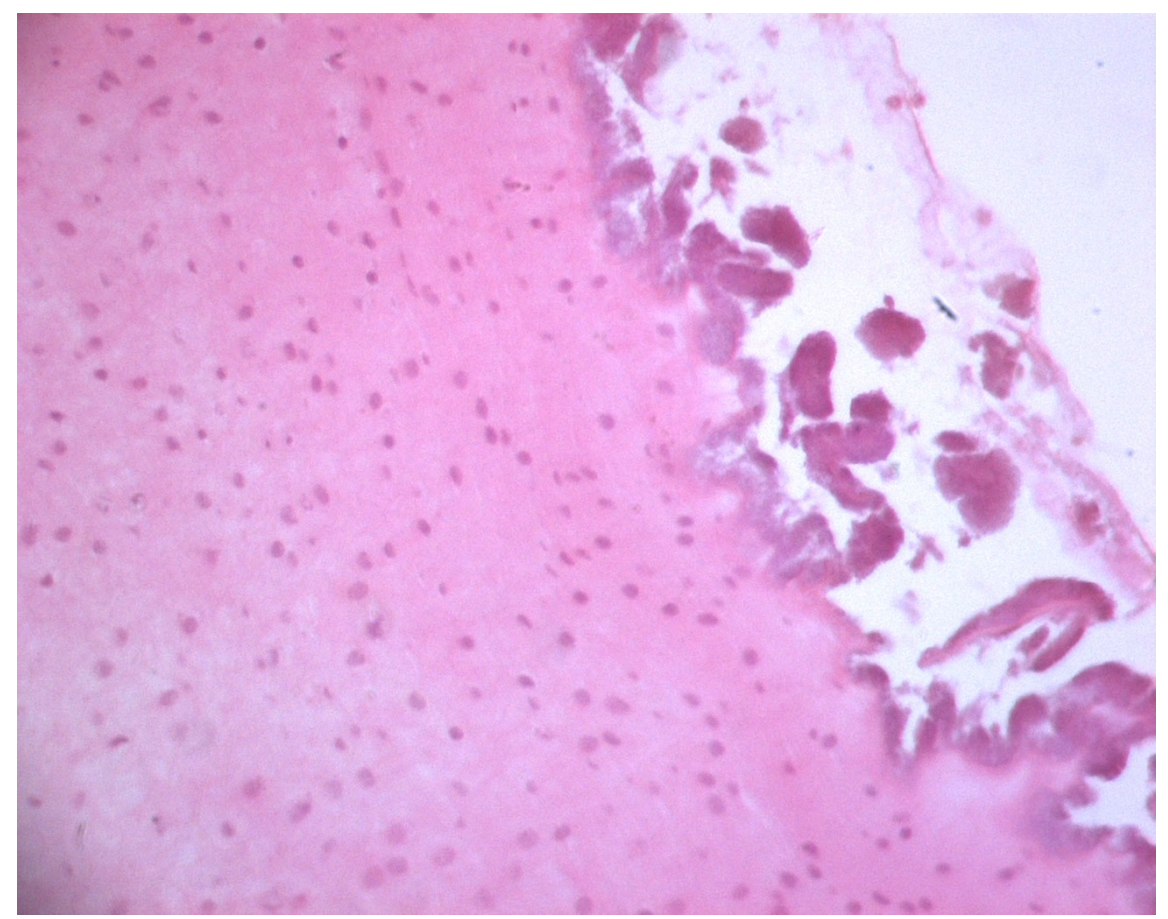

All the authors declare not having any conflicts of interest.

\section{Ethics statement:}

Data from the patient included in this case report were treated anonymously and a statement of informed consent was signed to allow the use of her medical and dental records and photos.

\section{Funding:}

The authors received no financial support for the research, authorship, and/or publication of this article.

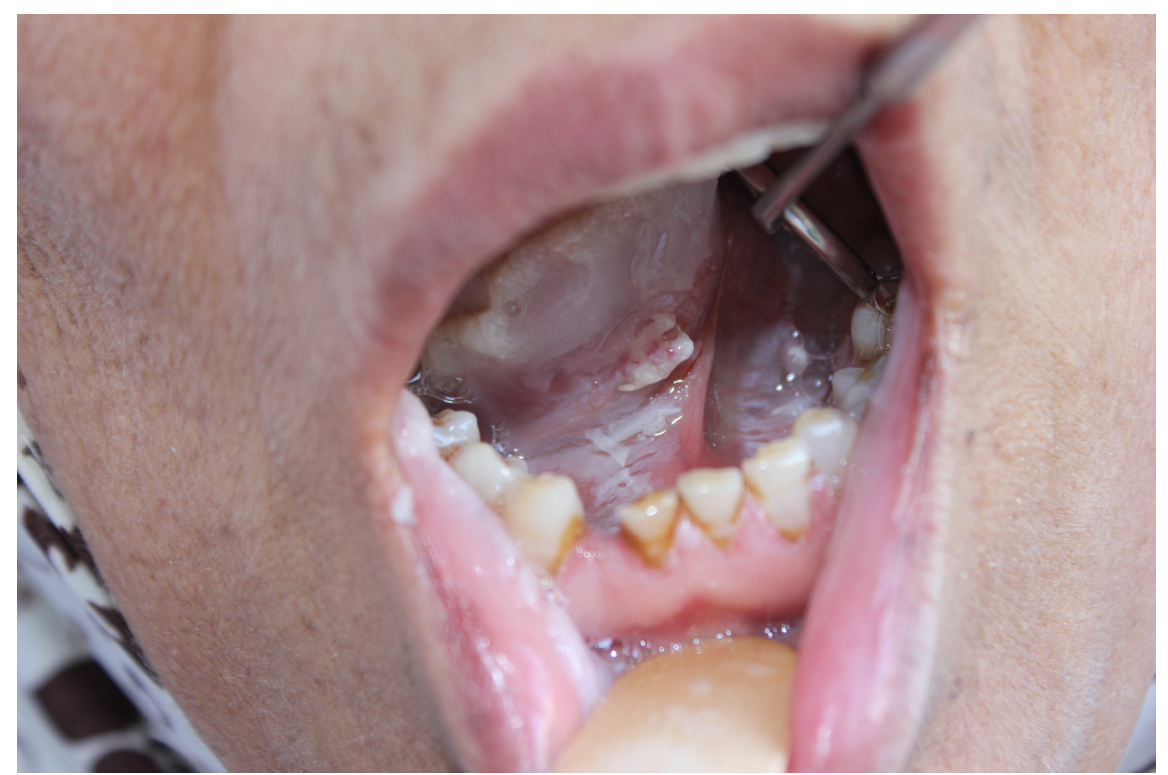



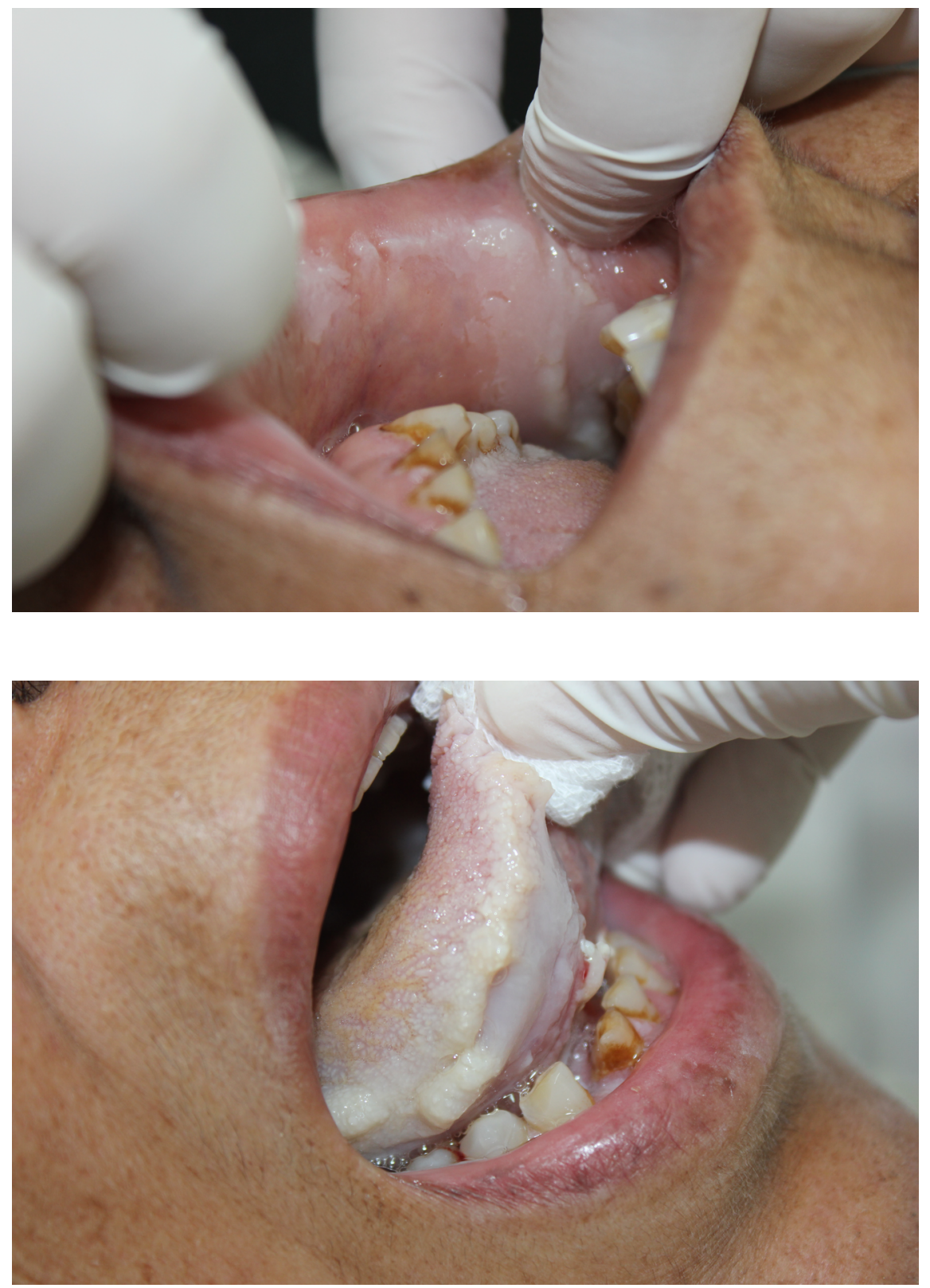


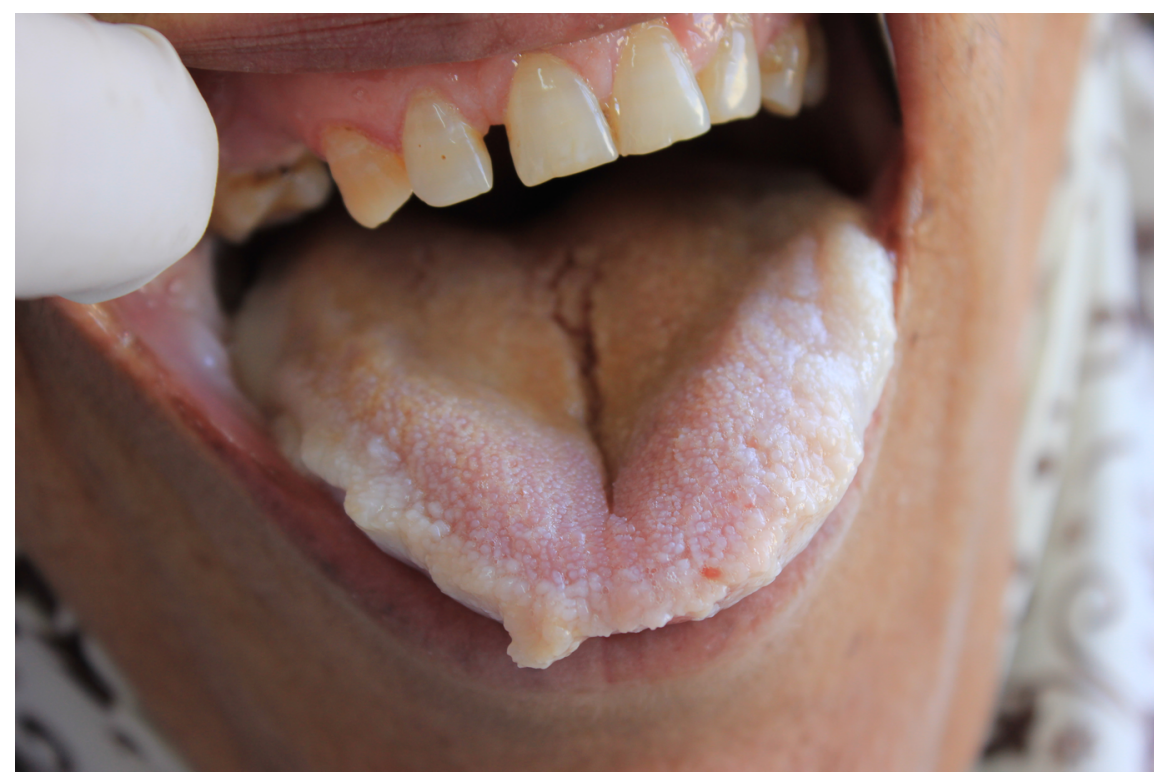

